\title{
Comparison of Intravesical Hyaluronic Acid, Chondroitin Sulfate, and Combination of Hyaluronic Acid-Chondroitin Sulfate Therapies in Animal Model of Interstitial Cystitis
}

\author{
Yavuz Onur Danacioglu ${ }^{1}$, Bulent Erol ${ }^{1}$, Seyma Ozkanli², Asif Yildirim ${ }^{1}$, Ramazan Gokhan Atis ${ }^{1}$, Mesrur Selcuk Silay ${ }^{1}$, \\ Turhan Caskurlu ${ }^{1}$ \\ ${ }^{1}$ Department of Urology, Istanbul Medeniyet University Faculty of Medicine, Goztepe Training \& Research Hospital, Istanbul, Turkey \\ ${ }^{2}$ Department of Pathology, Istanbul Medeniyet University Faculty of Medicine, Goztepe Training \& Research Hospital, Istanbul, Turkey
}

Purpose: Three intravesical treatment agents were compared in an interstitial cystitis rat model: chondroitin sulfate, hyaluronic acid, and combined hyaluronic acid-chondroitin sulfate.

Methods: Thirty-five female rats were divided into 5 groups: control (group I), isotonic (group II), chondroitin sulfate (group III), hyaluronic acid (group IV), and hyaluronic acid-chondroitin sulfate (group V). Chemical cystitis was induced in all experimental groups by intravesical instillation of $1 \mathrm{~mL}$ of hydrogen peroxide $\left(\mathrm{H}_{2} \mathrm{O}_{2}\right)$ for 15 minutes via the transurethral route. The treatment was administered every other day for 3 sessions 2 days after inducing chemical cystitis. Groups II, III, IV, and V received $1 \mathrm{~mL}$ of $0.9 \% \mathrm{NaCl}, 1 \mathrm{~mL}$ of $0.2 \%$ sodium chondroitin sulfate, $1 \mathrm{~mL}$ of low-molecular-weight hyaluronic acid, and 1 $\mathrm{mL}$ of $2 \%$ sodium chondroitin sulfate $+1.6 \%$ sodium hyaluronic acid, respectively. On day 7 , the animals were sacrificed and the bladders were removed for histopathological and immunohistochemical assessments.

Results: Significant between-group differences were found in vascular congestion $(\mathrm{P}=0.006)$. The grade of submucosal edema in groups II and IV was significantly higher than in group I $(\mathrm{P}=0.006, \mathrm{P}=0.006$, respectively). In group I, the grade of granulation tissue was lower than the other 4 groups, but no significant difference was found between the remaining groups $(\mathrm{P}=0.016)$. Neutrophil cell infiltration was more intense in groups II and IV than in group I $(\mathrm{P}=0.006, \mathrm{P}=0.006$, respectively). Significant differences in the leukocyte and mast cell count were detected between groups II and IV (P<0.001, P $<0.001$, respectively). Abnormal zonula occludens-1 and uroplakin-III immunoreactivity in group II was higher than in groups I, III, or $\mathrm{V}(\mathrm{P}=0.002, \mathrm{P}=0.010$, respectively). Interleukin-8 expression was lower in group $\mathrm{V}$ than in group II $(\mathrm{P}=0.001)$.

Conclusion: A single treatment of chondroitin sulfate and combined hyaluronic acid-chondroitin sulfate treatment demonstrated efficacy by suppressing inflammation and achieving improvements in the urothelium.

Keywords: Disease models, Animal; Inflammation; Cystitis, Interstitial; Rats; Therapeutic use

- Research Ethics: The approval of the study was received from the Local Ethics Committee (2017/591). All experimental protocols and procedures were approved by Yeditepe University Institutional Animal Care and Use Committee.

- Conflict of Interest: No potential conflict of interest relevant to this article was reported.

\section{- HIGHLIGHTS}

- This is the first study to evaluate the effectiveness of 3 different intravesical agents more clearly by performing both immunohistochemical and histopathological examinations.

- The single treatment of chondroitin sulfate and combined hyaluronic acid-chondroitin sulfate provided treatment efficacy by suppressing the earlystage inflammatory process in interstitial cystitis and achieving improvements in the urothelium.

- We demonstrated the comparable efficacy of chondroitin sulfate with combined hyaluronic acid-chondroitin sulfate in the present study.

Corresponding author: Bulent Erol (D) https://orcid.org/0000-0001-7099-6374 Department of Urology, Istanbul Medeniyet University Faculty of Medicine, Goztepe Training \& Research Hospital, 34700, Istanbul, Turkey

Email: erolbulent@yahoo.com

Submitted: September 21, 2019 / Accepted after revision: October 18, 2020 (c) (1) This is an Open Access article distributed under the terms of the Creative Commons Attribution Non-Commercial License (https://creativecommons.org/licenses/by-nc/4.0/) which permits unrestricted non-commercial use, distribution, and reproduction in any medium, provided the original work is properly cited. 


\section{INTRODUCTION}

Interstitial cystitis (IC) is a chronic disease of the urinary bladder induced by several etiological factors and characterized by urgency of urination, frequency of urination, nocturia, and pelvic pain. The morbidity of IC may vary depending on age, sex, and race according to prior epidemiological studies [1,2]. Even though this disease affects both sexes, especially within the age range of 30-50 years, approximately $90 \%$ of patients have been reported to be female. Although there is an ongoing debate regarding its etiology, it is believed that several etiological factors participate in the pathogenesis of IC, and disruption of the glycosaminoglycan (GAG) layer in the urothelial mucosa is the main histopathological factor leading to this clinical entity [3]. The GAG layer is composed of GAGs and proteoglycans such as chondroitin sulfate and hyaluronic acid [3,4]. Behavioral, oral and intravesical therapies, neuromodulation, and surgical treatment constitute the current therapeutic options for IC. Intravesical treatment with different agents, aiming to replace the disrupted GAG layer, represents the most commonly utilized invasive treatment method [5]. Replacement of the GAG layer as a treatment modality for IC has been preferred since the 1990s and was found to be more effective than other methods of IC treatment [6]. Physicians may experience difficulty in selection of the appropriate agent since no definitive outcome was obtained in various systematic reviews that analyzed the efficacy of different intravesical treatment agents. In this study, we aimed to compare the anti-inflammatory effects and regenerative capacities of intravesical instillation of chondroitin sulfate, hyaluronic acid, and a combination of hyaluronic acid-chondroitin sulfate agents in an experimental rat model of IC.

\section{MATERIALS AND METHODS}

\section{Animals and the Study Protocol}

Approval for the study was received from the local ethics committee (2017/591). All experimental protocols and procedures were approved by Yeditepe University Institutional Animal Care and Use Committee. The present study included 35 female Wistar albino rats aged between 4-6 months old and weighing between 200 and $250 \mathrm{~g}$. The experimental animals were given standard conditions, feeding, and maintenance during the sheltering. The rats were randomly divided into 5 experimental groups, each of which included 7 rats, as follows: control (group I), IC + isotonic solution (group II), IC + chondroitin sulfate (group III), IC + hyaluronic acid (group IV), IC + hyaluronic acid-chondroitin sulfate (group V). Chemical cystitis was induced in all groups by intravesical instillation of hydrogen peroxide $\left(\mathrm{H}_{2} \mathrm{O}_{2}\right)$ via the transurethral route, except in the sham control group with minor modifications [7]. Rats were anesthetized using $2 \%-3 \%$ isoflurane in $100 \%$ oxygen via a precision vaporizer. For the procedure, a sterile $26-\mathrm{G}$ catheter (Boston Scientific, Marlborough, MA, USA) was introduced into the urinary bladder via the transurethral route and the abdomen was pushed gently until the urine was removed. Then, $1 \mathrm{~mL}$ of $5 \% \mathrm{H}_{2} \mathrm{O}_{2}$ (Fisher Scientific, Hampton, NH, USA) was instilled via the transurethral route and held there for 15 minutes [7]. Respiratory rate, heart rate, and responses to painful stimuli were monitored in order to assess anesthetic depth in the rats during the experiment. Following the formation of chemical cystitis, the urinary bladder of each rat was drained by pressing the abdomen. One rat each from groups IV and V died due to impaired general condition and development of peritonitis after administration of $\mathrm{H}_{2} \mathrm{O}_{2}$.

\section{Treatment Protocol}

The treatment protocol was administered every other day for a total of 3 sessions in all of the experimental groups (i.e., excluding group I) 2 days after the formation of chemical cystitis.

Group I $(n=7)$ : This healthy group of animals, which were maintained in the same conditions without induction of chemical cystitis, was sacrificed and underwent cystectomy concurrently with the other rats. Excised specimens were processed for conventional histopathological examination.

Group II $(n=7)$ : The animals in this group were instilled with $1 \mathrm{~mL}$ of $0.9 \% \mathrm{NaCl}$ via the transurethral route every other day for a total of 3 sessions using a $26-\mathrm{G}$ purple angiocath.

Group III ( $\mathrm{n}=7)$ : In this group, $1 \mathrm{~mL}$ of $0.2 \%$ sodium chondroitin sulfate (Gepan, Instill, Farmatek, Istanbul, Turkey) was instilled via the transurethral route using a $26-\mathrm{G}$ purple angiocath every other day for a total of 3 sessions [8].

Group IV ( $\mathrm{n}=6)$ : In this group, $1 \mathrm{~mL}$ of low-molecular-weight hyaluronic acid (120 mg of hyaluronic acid in $50 \mathrm{~mL}$ ) (Hyacyst, Instill, Syner-Med, Surrey, England) was instilled via the transurethral route using a $26-\mathrm{G}$ purple angiocath every other day for a total of 3 sessions.

Group V ( $\mathrm{n}=6)$ : In this group, $1 \mathrm{~mL}$ of $2 \%$ sodium chondroitin sulfate $+1.6 \%$ sodium hyaluronic acid (Ialuril Instill, IBSA, Lugano, Sweden) was instilled via the transurethral route using a 26-G purple angiocath every other day for a total of 3e ses- 
sions. The solutions were maintained for 1 hour in the bladder of each animal. The bladders were emptied by pushing the abdomen after 1 hour in all groups except for group I.

Animals were sacrificed using $200 \mathrm{mg} / \mathrm{kg}$ of pentobarbital 1 day after the third session of the treatment, and their bladders were removed to perform histopathological and immunohistochemical examinations.

\section{Histopathological Evaluation}

Urinary bladders were fixed in $10 \%$ neutral buffered formalin after removal and embedded in paraffin. Three micron sections were obtained from the paraffin-embedded tissues using a rotary microtome and stained with hematoxylin and eosin per standard procedures. The examinations were performed using a light microscope (BX-51, Olympus, Tokyo, Japan) and an Olympus DP72 camera system. The histopathological assessments were based on the following classification for each therapeutic group. The histological slides were analyzed by a blinded pathologist who received the specimens identified with only numbers without a label indicating the groups. The severity of inflammation was evaluated at $\times 10(\times 10$ optical zoom $)$ magnification; vascular congestion, submucosal edema, mononuclear lymphoid cells, grade of granulation, and neutrophil infiltration were evaluated and scored in 10 different fields of the sections for each sample. The specimens with no submucosal edema, mild submucosal edema (without a change in the thickness of connective tissue), moderate submucosal edema (if thickness of the connective tissue increased less than 2-fold), and severe submucosal edema (if thickness of the connective tissue increased more than 2-fold) were scored as $0,1,2$, and 3 , respectively. Specimens with high levels of mononuclear lymphoid cells, vascular congestion, granulation, and leukocyte accumulation were scored as $0,1,2$, and 3 , respectively. Leukocytes were counted by assessing each specimen in 10 different identical fields at $\times 40(\times 40$ optical zoom $)$ magnification. Mast cells were counted by staining the mast cells using a mast cell tryptase antibody (Leica Biosystems, Wetzlar, Germany) and assessing each specimen in 10 different identical fields at $\times 40$ magnification. Total values of infiltration scores for leukocytes and mast cells were calculated separately for each group.

\section{Immunohistochemical Evaluation}

The grade of epithelial integrity and epithelial damage was assessed by the uroplakin-III (UP-III) polyclonal antibody, the Ecadherin monoclonal antibody (Cell Marque, Rocklin, CA, USA), and the zonula occludens-1 (ZO-1) polyclonal antibody (Thermo Scientific, Waltham, MA, USA), while the severity of inflammation was evaluated by the interleukin-8 (IL-8) antibody (Novus Biologicals, Centennial, CO, USA) with an immunohistochemical evaluation. Each specimen was assessed separately at $\times 40$ magnification. The assessment was carried out using an Olympus BX51 light microscope and an Olympus DP72 digital camera system. Normal expression of UP-III, Ecadherin, and ZO-1 was defined as even distribution throughout the urothelium, mainly in the surface of the epithelial cells. Abnormal expression was defined as weak expression and distribution throughout the urothelium. Specimens with no IL-8 expression, IL-8 expression with mild vascular intensity, IL-8 expression with moderate vascular intensity, and IL-8 expression with severe vascular intensity were scored as $0,1,2$, and 3 , respectively.

\section{Statistical Analysis}

IBM SPSS Statistics ver. 22.0 (IBM Co., Armonk, NY, USA) was used for all statistical analyses. The chi-square test was used to compare the results of histological analyses between groups. A post hoc analysis was performed for comparisons of columns for proportional analyses, and the Bonferroni correction was applied to the P-values. The Kruskal-Wallis test was used to compare leukocyte and mast cell counts between the groups. A pairwise comparison was performed for post hoc analysis, and the Bonferroni correction was applied to the P-values. P-values less than 0.05 were considered to indicate statistical significance.

\section{RESULTS}

\section{Histopathological Analysis}

A significant difference was found between groups I and IV in vascular congestion $(\mathrm{P}=0.006)$. However, no significant difference was found between the other groups in this parameter. The grades of submucosal edema were significantly higher in groups II and IV than in group I $(\mathrm{P}=0.006$ and $\mathrm{P}=0.006$, respectively). The intensity of mononuclear lymphoid cell infiltration was significantly lower in group I than in the other groups (i.e., groups II, III, IV, and V) $(\mathrm{P}=0.006, \mathrm{P}=0.046, \mathrm{P}=0.006$, and $\mathrm{P}=0.046$, respectively). The grade of granulation tissue formation was significantly lower in group I than in the other 4 groups; however, there was no statistically significant difference between those groups $(\mathrm{P}=0.016)$. The intensity of neutrophil 
cell infiltration was significantly higher in groups II and IV than in group $\mathrm{I}(\mathrm{P}=0.006$ and $\mathrm{P}=0.006$, respectively) (Figs. 1,2$)$.

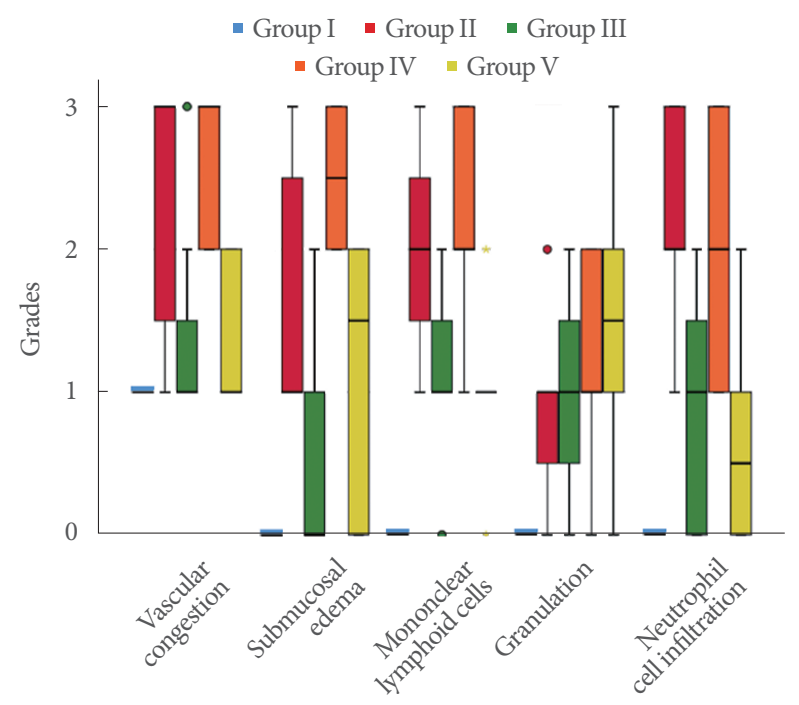

Fig. 1. Clustered boxplot of grades by histopathological results by group. Group I, control; group II, interstitial cystitis + isotonic solution; group III, interstitial cystitis + chondroitin sulfate; group IV, interstitial cystitis + hyaluronic acid; group V, interstitial cystitis + hyaluronic acid + chondroitin sulfate.
Significant between-group differences in the leukocyte count were found between groups I and IV, groups I and II, and groups II and $\mathrm{V}(\mathrm{P}=0.004, \mathrm{P}<0.001$, and $\mathrm{P}=0.004$, respectively). Significant differences in the mast cell count were found between groups I and IV, groups I and II, groups II and V, and groups IV and $\mathrm{V}(\mathrm{P}=0.005, \mathrm{P}<0.001, \mathrm{P}=0.042$, and $\mathrm{P}=0.005$, respectively) (Figs. 3, 4).

\section{Immunohistochemical Analysis}

Group II had a significantly higher rate of abnormal expression of ZO-1 and UP-III than groups I, III, and V. Group IV showed significantly higher rates of abnormal expression of ZO- 1 and UP-III than group I ( $\mathrm{P}=0.002$ and $\mathrm{P}=0.010$, respectively). No significant between-group differences were found in abnormal E-cadherin expression, although the level of IL-8 expression was significantly lower in group I than in groups II, III, and IV $(\mathrm{P}=0.562$ and $\mathrm{P}=0.001$, respectively). Group $\mathrm{V}$ also had a significantly lower level of IL-8 expression than group II ( $\mathrm{P}=0.001)$ (Table 1; Figs. 4, 5).

\section{DISCUSSION}

In this study, we investigated inflammatory process in the uro-
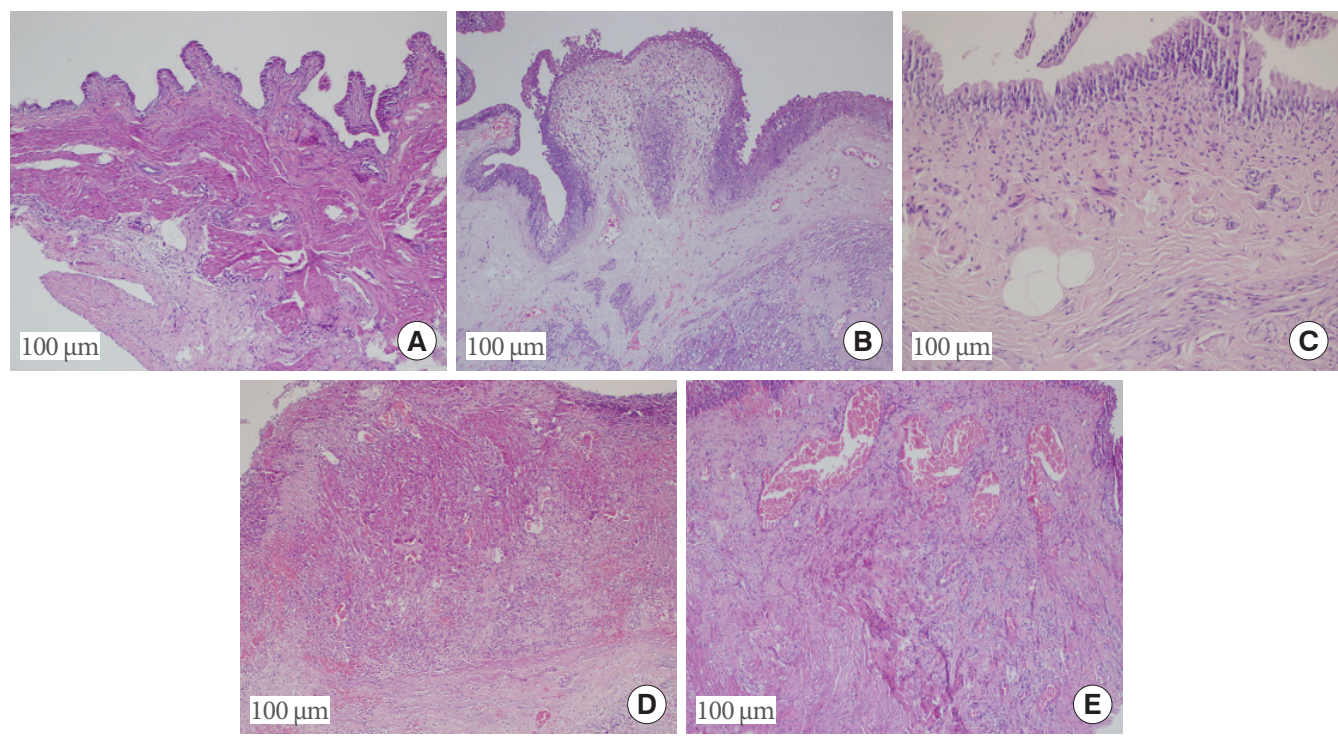

Fig. 2. (A) Full-thickness section of the normal bladder in group I: normal urothelium, submucosa, and muscularis propria (hematoxylin and eosin $[\mathrm{H} \& \mathrm{E}], \times 10$ ). (B) In group II: severe vascular congestion and submucosal edema accompanied by severe neutrophilic and mononuclear infiltration $(\mathrm{H} \& \mathrm{E}, \times 10)$. (C) In group III: mild vascular congestion accompanied by moderate neutrophilic and severe mononuclear infiltration $(\mathrm{H} \& \mathrm{E}, \times 10)$. (D) In group IV: severe vascular congestion and submucosal edema accompanied by severe neutrophilic and mononuclear infiltration and granulation tissue $(H \& E, \times 10)$. (E) In group V: moderate vascular congestion accompanied by mild neutrophilic and severe mononuclear infiltration and granulation tissue $(\mathrm{H} \& \mathrm{E}, \times 10)$. 
thelium in a rat IC model and analyzed the recovery process achieved by the therapies. This is the first study that has compared the histopathological and immunohistochemical results achieved by 3 different intravesical agents in the treatment of IC. Urinary solutes never contact the urothelium during the filling phase of the bladder due to umbrella cells. In addition, GAG and other proteoglycans in the umbrella cells protect the permeability of the urothelium. A decrease in the permeability

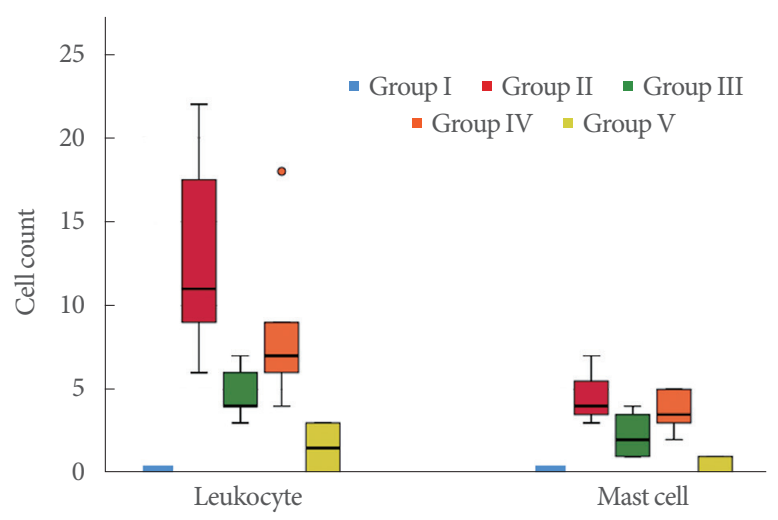

Fig. 3. Clustered boxplot of leukocyte and mast cell counts by group. Group I, control; group II, interstitial cystitis + isotonic solution; group III, interstitial cystitis + chondroitin sulfate; group IV, interstitial cystitis + hyaluronic acid; group V, interstitial cystitis + hyaluronic acid + chondroitin sulfate. function of the umbrella cells due to various factors may lead to IC $[9,10]$. It has been shown that in the pathogenesis of IC, pain associated with nerve depolarization occurs after the diffusion of urea, calcium, and potassium through the urothelium, which exert toxic effects on the urothelium by disrupting the epithelial permeability barrier on the bladder surface [9]. GAG replacement agents such as hyaluronic acid, heparin sulfate, heparin, chondroitin sulfate, and keratan sulfate are currently preferred as treatment options for IC [8].

In the literature, various rat models that can mimic IC by creating inflammation of the bladder have been described. No animal model can be expected to reflect all the symptoms experienced by patients, and it is obvious that all aspects of IC cannot be mimicked by a single model. Rat loss may occur during the experiment, or optimal inflammation may be not obtained in various animal models described in the literature [11]. However, in the present study, we induced a long-lasting cystitis model using $\mathrm{H}_{2} \mathrm{O}_{2}$ as presented by Dogishi et al. [7], rather than other intravesical or intraperitoneal agents, considering the high regenerative capacity of the rat bladder [12]. In our study, sufficient chemical cystitis could be created and only 2 rats died during the experiment. We demonstrated early-stage histological changes and alteration of the antigens that are responsible for permeability in the urothelium, which is consistent with the
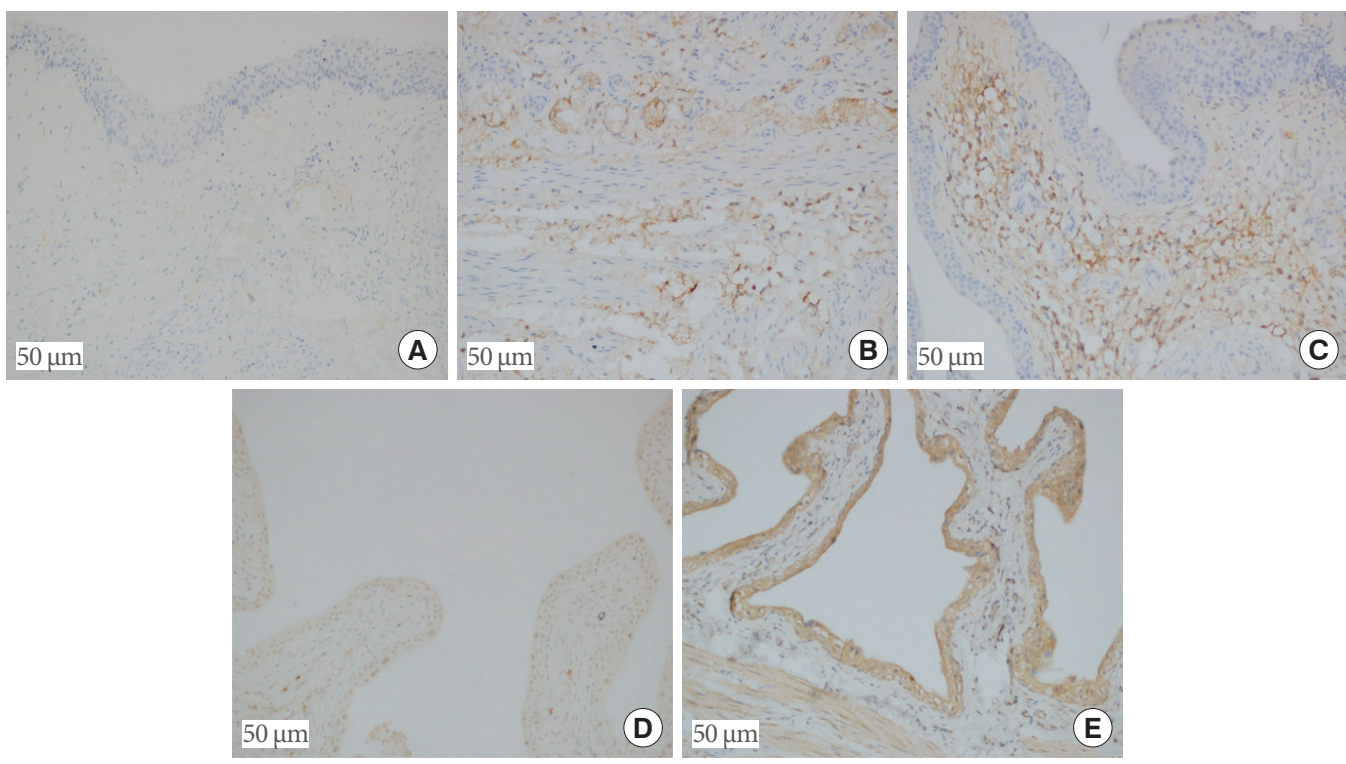

Fig. 4. (A) Absence of mast cells, confirmed by immunohistochemistry (mast cell tryptase, $\times 20$ ). (B) A small number of mast cells in the submucosa of group III (mast cell tryptase, $\times 20$ ). (C) Increased number of mast cells in the submucosa of group II (mast cell tryptase, $\times 20$ ). (D) ZO-1 positivity seen immunohistochemically in the urothelial epithelium in group V $(\mathrm{ZO}-1, \times 20)$. (E) Fullthickness immunohistochemical ZO-1 positivity in the urothelial epithelium in group IV $(\mathrm{ZO}-1, \times 20)$. 
literature. Engles et al. [13] demonstrated the therapeutic effect of chondroitin sulfate on the inflammatory process. Our study showed that mononuclear cells and neutrophil infiltration, ac- cepted as the ideal parameters for evaluation of inflammation, were significantly lower in group $\mathrm{V}$ than in group IV and group II [7]. In our study, treatment of chondroitin sulfate and com-

Table 1. Distribution of the immunohistochemical results of the measurements by group

\begin{tabular}{|c|c|c|c|c|c|c|}
\hline Variable & Group I (n=7) & Group II $(\mathrm{n}=7)$ & Group III $(n=7)$ & Group IV $(n=6)$ & Group V $(n=6)$ & P-value \\
\hline $\mathrm{ZO}-1$ & & & & & & 0.002 \\
\hline Abnormal & $0(0)$ & $7(100)$ & $3(42.9)$ & $5(83.3)$ & $2(33.3)$ & \\
\hline Normal & $7(100)$ & $0(0)$ & $4(57.1)$ & $1(16.7)$ & $4(66.7)$ & \\
\hline UP- III & & & & & & 0.010 \\
\hline Abnormal & $1(14.3)$ & $6(85.7)$ & $2(28.6)$ & $5(83.3)$ & $1(16.7)$ & \\
\hline Normal & $6(85.7)$ & $1(14.3)$ & $5(71.4)$ & $1(16.7)$ & $5(83.3)$ & \\
\hline E-cadherin & & & & & & 0.562 \\
\hline Abnormal & $7(100)$ & $6(85.7)$ & $5(71.4)$ & $4(66.7)$ & $5(83.3)$ & \\
\hline Normal & $0(0)$ & $1(14.3)$ & $2(28.6)$ & $2(33.3)$ & $1(16.7)$ & \\
\hline IL-8 level & & & & & & 0.001 \\
\hline 0 & $6(85.7)$ & $0(0)$ & $1(14.3)$ & $0(0)$ & $1(16.7)$ & \\
\hline 1 & $1(14.3)$ & $0(0)$ & $2(28.6)$ & $0(0)$ & $4(66.7)$ & \\
\hline 2 & $0(0)$ & $3(42.9)$ & $3(42.9)$ & $3(50)$ & $1(16.7)$ & \\
\hline 3 & $0(0)$ & $4(57.1)$ & $1(14.3)$ & $3(50)$ & $0(0)$ & \\
\hline
\end{tabular}

Values are presented as number (\%).

ZO-1, zonula occludens-1; UP- III, uroplakin- III; IL-8, interleukin-8; Group I, control; Group II, interstitial cystitis + isotonic solution group; Group III, interstitial cystitis + chondroitin sulfate; Group IV, interstitial cystitis + hyaluronic acid; Group V, interstitial cystitis + hyaluronic acid + chondroitin sulfate.
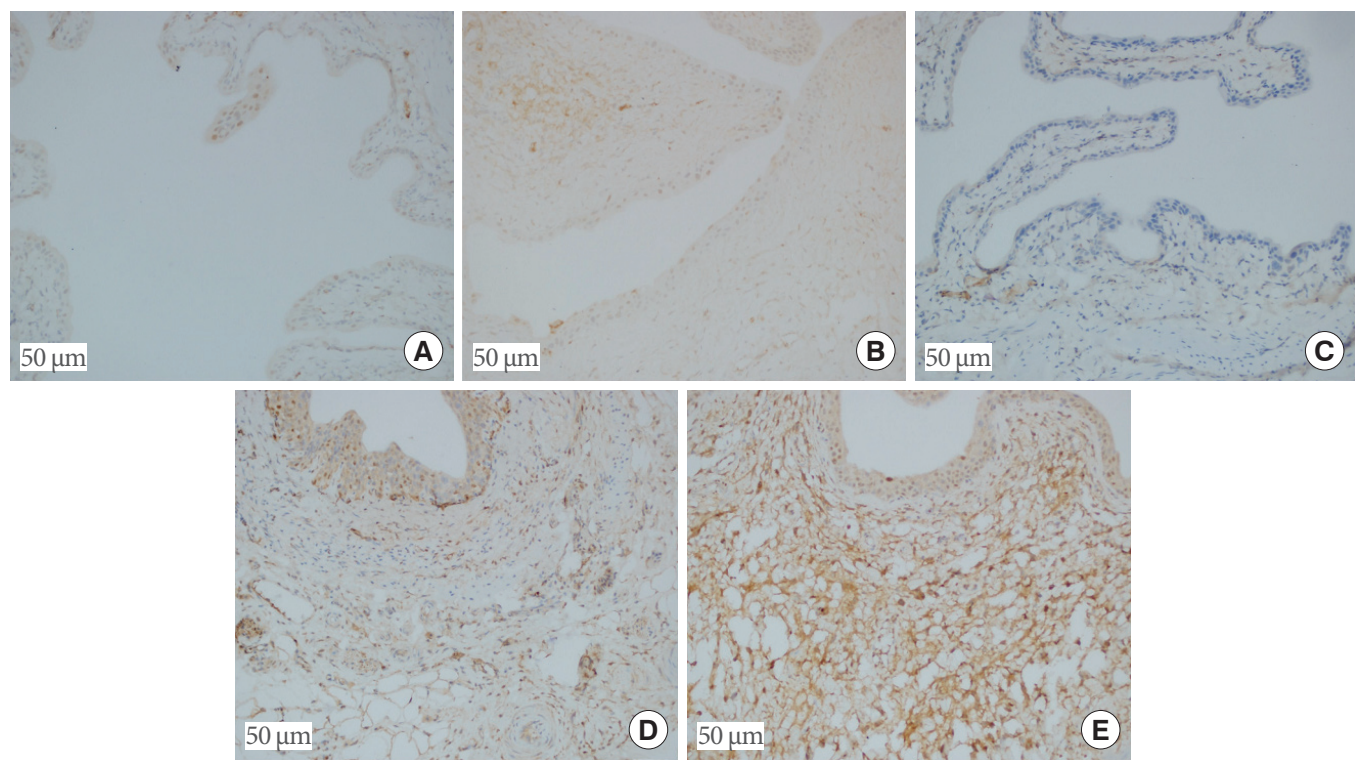

Fig. 5. (A) Positive immunoreactivity with UP-III in the urothelium in group III (uroplakin, $\times 20$ ). (B) Negative immunoreactivity with UP-III in the urothelium in group II (uroplakin, $\times 20$ ). (C) No expression of IL-8 in group I (IL-8, $\times 20$ ). (D) Moderate IL-8 expression in the mucosa and the submucosa of group V (IL-8, $\times 20)$. (E) Strong IL-8 expression in the mucosa and the submucosa of group IV (IL-8, ×20). UP-III, uroplakin; IL, interleukin. 
bined hyaluronic acid-chondroitin sulfate suppressed the earlystage inflammatory process in IC, aligning with findings in the literature. The intensity of granulation tissue is an early-stage indicator of improvement in damaged tissues [14]. We found that granulation tissue intensity in the control group was lower than in the other 4 groups. However, no statistically significant difference was found between those 4 groups. This finding demonstrates that urinary bladders of rats have a high regenerative capacity with or without treatment. This finding also provides useful information about the duration of the experimental animal models for further studies.

An increased number of leukocytes and mast cells has been shown as an indicator of acute inflammation in many experimental IC models, and treatment response has been shown by a reduced number of these cells $[15,16]$. Mast cells, which play an important role in the pathophysiology of IC, lead to the release of various nociceptive mediators, and quantities of neuropeptide $\mathrm{Y}$ and substance-P-positive sympathetic nerves increase for this reason in patients with IC. The relationship between increased quantities of nerve fibers in the bladder and mast cells has been proven in various experimental animal models. Studies have shown that symptoms of inflammatory diseases such as IC and irritable bowel syndrome might worsen due to activation of mast cells and nerve fibers [17,18]. Sinanoglu et al. [8] detected in an experimental rat model that treatment with chondroitin sulfate provided a significant reduction in leukocyte and mast cell counts in the urothelium and restored the GAG layer. The leukocyte and mast cell counts also significantly decreased in groups III, IV, and V in our study. This anti-inflammatory effect linked to intravesical treatments can provide regression in symptoms by suppressing the anti-inflammatory process and reducing mast cell count. Increased levels of IL-8 have been encountered in the urine of IC patients [19]. Previous studies reported that this increase was not correlated with patients' symptoms, but rather with mast cell infiltration in the urothelium [20]. This suggests that IL-8 functions as a key chemokine for mast cells and that mast cells stimulate the urothelium to release IL-8 [21,22]. Rooney et al. [19] presented in their in vitro IC model that IL-8 expression decreased due to treatment with hyaluronic acid and consequently caused a regression in the inflammation of the urothelium by reducing mast cell infiltration. We also detected that the level of IL- 8 decreased in all treatment groups, with the most significant reduction achieved in group V. In addition, group V showed the highest reduction in the mast cell count. This finding once more shows the correlation between IL-8 expression and mast cell infiltration, as supported by the literature.

ZO-1 is a ubiquitous component of tight junction proteins located in the urothelial epithelium, which maintain the permeability barrier of the urothelium. ZO-1 is expressed by the apicolateral part of superficial umbrella cells [23]. ZO-1 has been accepted as a specific and sensitive biomarker for IC patients [24]. In a ketamine-induced IC model, Duan et al. [25] detected that the abnormal distribution of ZO-1 proteins after 8 weeks was $90 \%$, as opposed to $0 \%$ in the control group. We showed that abnormal expression of ZO-1 was significantly higher in group II than in groups I, III, and V.

Uroplakin is a mammalian protein synthesized in the superficial urothelial epithelium. The uroplakin proteins are highly conserved, transmembrane proteins that function as key components in mechanical stabilization of the urothelial surface [26]. In an experimental rat model, Lv et al. [27] found that the urothelium was thinner and that abnormal expression of UPIII increased. It was observed that expression of UP-III was close to its normal level in rats that were treated with intravesical hyaluronic acid. In our study, we found that the highest abnormal expression of UP-III was in group II. Normal UP-III expression increased in groups III and V.

E-cadherin is a urothelial adhesion protein, reduced expression of which leads to urothelial barrier dysfunction. Therefore, it has been used as a biomarker in several IC model studies. Li et al. [28], who induced ketamine-induced cystitis, performed an evaluation of E-cadherin expression using a monoclonal antibody in their experimental study. We used a similar method in our study. However, no statistically significant difference was found between the groups regarding abnormal expression of Ecadherin.

The biomarkers mentioned in our study have been evaluated individually in various other studies. This is the first study to evaluate the effectiveness of intravesical agents more clearly by performing both immunohistochemical and histopathological examinations.

There are several opinions about the clinical use and outcomes of intravesical agents, and there is currently no consensus on the optimal instillation regimens of intravesical agents [29]. The superiority of intravesical treatments with chondroitin sulfate and combined hyaluronic acid-chondroitin sulfate compared with other treatment models in reducing inflammation and achieving epithelial improvement was shown considering all biomarkers assessed in this study. We also demonstrated the 
comparable efficacy of chondroitin sulfate with combined hyaluronic acid-chondroitin sulfate in the present study.

There are some limitations of our study. First, the efficacy of treatments performed in the rat bladder with a high regenerative capacity may present differences compared with the human bladder. The treatment was evaluated based on the early-term findings of the histological and immunohistochemical analyses in this study. However, IC is a chronic and symptomatic disease and should be evaluated with histological and cytometric assessments of long-term treatment.

In conclusion, our experimental study is the first analysis to compare the histopathological and immunohistochemical findings of 3 different intravesical treatment agents. We have shown that single treatment of chondroitin sulfate and combined hyaluronic acid-chondroitin sulfate provided treatment efficacy by suppressing the inflammatory process and achieving improvement in the urothelium. Further studies are needed to clarify the efficacy of different agents in IC treatment.

\section{AUTHOR ORCID}

$\begin{array}{ll}\text { Yavuz Onur Danacioglu } & 0000-0002-3170-062 X \\ \text { Bulent Erol } & 0000-0003-2402-3373 \\ \text { Seyma Ozkanli } & 0000-0002-3357-1059 \\ \text { Asif Yildirim } & 0000-0002-3386-971 X \\ \text { Ramazan Gokhan Atis } & 0000-0002-9065-6104 \\ \text { Mesrur Selcuk Silay } & 0000-0001-5091-9654 \\ \text { Turhan Caskurlu } & 0000-0002-4471-2670\end{array}$

\section{AUTHOR CONTRIBUTION STATEMENT}

- Conceptualization: BE, TC, AY

- Data curation: YOD, SO, RGA

- Formal analysis: BE, TC, AY

- Funding acquisition: MSS, YOD, RGA

- Methodology: BE, YOD, MSS

- Project administration: BE, TC, YOD

- Visualization: SO, BE, AY, RGA

-Writing-original draft: YOD, BE, TC

- Writing-review \& editing: BE, YOD, TC

\section{REFERENCES}

1. Hanno PM. Diagnosis of interstitial cystitis. Urol Clin North Am 1994;21:63-6.
2. Chuang YC, Chermansky C, Kashyap M, Tyagi P. Investigational drugs for bladder pain syndrome (BPS) / interstitial cystitis (IC). Expert Opin Investig Drugs 2016;25:521-9.

3. Pyo JS, Cho WJ. Systematic review and meta-analysis of intravesical hyaluronic acid and hyaluronic acid/chondroitin sulfate instillation for interstitial cystitis/painful bladder syndrome. Cell Physiol Biochem 2016;39:1618-25.

4. Hurst RE, Roy JB, Min KW, Veltri RW, Marley G, Patton K, et al. A deficit of chondroitin sulfate proteoglycans on the bladder uroepithelium in interstitial cystitis. Urology 1996;48:817-21.

5. Matsuoka PK, Haddad JM, Pacetta AM, Baracat EC. Intravesical treatment of painful bladder syndrome: a systematic review and meta-analysis. Int Urogynecol J 2012;23:1147-53.

6. Kurth K, Parsons CL. The interstitial cystitis syndrome: intravesical and oral treatment. Eur Urol Suppl 2003;2:2-9.

7. Dogishi K, Okamoto K, Majima T, Konishi-Shiotsu S, Homan T, Kodera M, et al. A rat long-lasting cystitis model induced by intravesical injection of hydrogen peroxide. Physiol Rep 2017;5:e13127.

8. Sinanoglu O, Dogan Ekici I, Ekici S. Comparison of intravesical application of chondroitin sulphate and colchicine in rat protamine/lipopolysaccharide induced cystitis model. Urol J 2014;11: 1296-300.

9. Parsons CL. The role of the urinary epithelium in the pathogenesis of interstitial cystitis/prostatitis/urethritis. Urology 2007;69(4 Suppl):9-16.

10. Huygens A, Crnolatac I, Maes J, Van Cleynenbreugel B, Van Poppel $\mathrm{H}$, Roskams T, et al. Influence of the glycosaminoglycan layer on the permeation of hypericin in rat bladders in vivo. BJU Int 2007;100: 1176-81.

11. Birder L, Andersson KE. Animal modelling of interstitial cystitis/ bladder pain syndrome. Int Neurourol J 2018;22(Suppl 1):S3-9.

12. Delos Santos GB, Devine MY, Wetterlin J, Firmiss PR, Kukulka NA, Bowen DK, et al. Compensatory regrowth of the mouse bladder after partial cystectomy. PLoS One 2018;13:e0206436.

13. Engles CD, Hauser PJ, Abdullah SN, Culkin DJ, Hurst RE. Intravesical chondroitin sulfate inhibits recruitment of inflammatory cells in an acute acid damage "leaky bladder" model of cystitis. Urology 2012;79:483.e13-7.

14. Bury MI, Fuller NJ, Meisner JW, Hofer MD, Webber MJ, Chow LW, et al. The promotion of functional urinary bladder regeneration using anti-inflammatory nanofibers. Biomaterials 2014;35: 9311-21.

15. Theoharides TC, Sant GR, el-Mansoury M, Letourneau R, Ucci AA Jr, Meares EM Jr. Activation of bladder mast cells in interstitial cystitis: a light and electron microscopic study. J Urol 1995;153(3 Pt 
1):629-36.

16. Bayrak O, Erturhan S, Seckiner I, Erbagci A, Ustun A, Karakok M. Chemical cystitis developed in experimental animals model: topical effect of intravesical ozone application to bladder. Urol Ann 2014;6:122-6.

17. Ercan F, Akici A, Ersoy Y, Hürdag C, Erin N. Inhibition of substance $\mathrm{P}$ activity prevents stress-induced bladder damage. Regul Pept 2006;133:82-9.

18. Farthing M. Irritable bowel, irritable body or irritable brain? Biomed Pharmacother 1995;6:312-3.

19. Rooney P, Srivastava A, Watson L, Quinlan LR, Pandit A. Hyaluronic acid decreases IL-6 and IL-8 secretion and permeability in an inflammatory model of interstitial cystitis. Acta Biomater 2015; 19:66-75.

20. Erickson DR, Tomaszewski JE, Kunselman AR, Stetter CM, Peters $\mathrm{KM}$, Rovner ES, et al. Urine markers do not predict biopsy findings or presence of bladder ulcers in interstitial cystitis/painful bladder syndrome. J Urol 2008;179:1850-6.

21. Valent P. Cytokines involved in growth and differentiation of human basophils and mast cells. Exp Dermatol 1995;4(4 Pt 2):255-9.

22. Batler RA, Sengupta S, Forrestal SG, Schaeffer AJ, Klumpp DJ. Mast cell activation triggers a urothelial inflammatory response mediated by tumor necrosis factor-alpha. J Urol 2002;168:819-25.
23. Lavelle J, Meyers S, Ramage R, Bastacky S, Doty D, Apodaca G, et al. Bladder permeability barrier: recovery from selective injury of surface epithelial cells. Am J Physiol Renal Physiol 2002;283:F24253.

24. Keay S. Cell signaling in interstitial cystitis/painful bladder syndrome. Cell Signal 2008;20:2174-9.

25. Duan Q, Wu T, Yi X, Liu L, Yan J, Lu Z. Changes to the bladder epithelial barrier are associated with ketamine-induced cystitis. Exp Ther Med 2017;14:2757-62.

26. Wu XR, Kong XP, Pellicer A, Kreibich G, Sun TT. Uroplakins in urothelial biology, function, and disease. Kidney Int 2009;75:115365.

27. Lv YS, Yao YS, Rong L, Lin ME, Deng BH, Xie Y, et al. Intravesical hyaluronidase causes chronic cystitis in a rat model: a potential model of bladder pain syndrome/interstitial cystitis. Int J Urol 2014;21:601-7.

28. Li M, Yang K, Wang X, Xu X, Zhu L, Wang H. Mast cells infiltration and decreased E-cadherin expression in ketamine-induced cystitis. Toxicol Rep 2014;2:205-9.

29. Zhang W, Deng X, Liu C, Wang X. Intravesical treatment for interstitial cystitis/painful bladder syndrome: a network meta-analysis. Int Urogynecol J 2017;28:515-25. 